\title{
Preoperative evaluation of patients with liver disease
}

\author{
A James Hanje and Tushar Patel \\ Nature Clinical Practice Gastroenterology \& Hepatology (2007) 4: 266-276 [doi:10.1038/ncpgasthep0794]
}

\section{ERRATUM}

In the May 2007 issue, in the Review by Hanje and Patel, there were errors in the second sentence of the section on 'Obstructive jaundice' on page 268. The correct sentence should read 'These risk factors include an initial hematocrit of less than $30 \%$, a serum total bilirubin concentration above $11 \mathrm{mg} / \mathrm{dl}$, the presence of malignancy, a serum creatinine concentration higher than $1.4 \mathrm{mg} / \mathrm{dl}$, serum albumin concentration lower than $3.0 \mathrm{~g} / \mathrm{dl}$, age older than 65 years, aspartate aminotransferase concentration above $90 \mathrm{lU} / \mathrm{l}$, and blood urea nitrogen concentration above $19 \mathrm{mg} / \mathrm{dl}^{18-20}$, doi:10.1038/ncpgasthep0873

\section{Don't miss the following articles that will soon be appearing in the pages of Nature Clinical Practice Gastroenterology \& Hepatology:}

\section{VIEWPOINT}

Screening and surveillance in Barrett's esophagus an unresolved dilemma

Stuart J Spechler

\section{PRACTICE POINTS}

Is antifibrinolytic drug use in liver transplantation associated with an increased risk of thromboembolic events?

Andre De Wolf and Michael Abecassis

Tacrolimus or microemulsified ciclosporin for immunosuppression in patients after liver transplantation?

Paul Y Kwo, Mubashir Khan and A Joseph Tector

Is there an increased risk of hip fracture in patients on long-term PPI therapy?

Nicholas J Talley, Hye Kyung Jung, Xuan Jiang and G Richard Locke

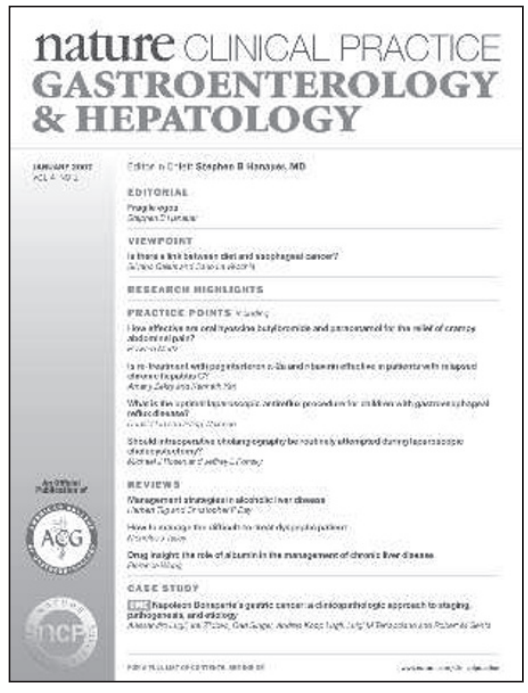

Is the rate of adenoma detection in colonoscopy influenced by the duration of colonoscope withdrawal? Paul D Collins and Alastair JM Watson

Unsedated small-caliber endoscopy-new screening tool for Barrett's esophagus?

Matthew Atkinson and Amitabh Chak

\section{REVIEW}

Evaluation and management of obesity-related nonalcoholic fatty liver disease Clare Nugent and Zobair M Younossi

Please note that the title details are not finalized and might be subject to change. 